\title{
Golgi and sarcolemmal neuronal NOS differentially regulate contraction-induced fatigue and vasoconstriction in exercising mouse skeletal muscle
}

\author{
Justin M. Percival, ${ }^{1}$ Kendra N.E. Anderson, ${ }^{1}$ Paul Huang, ${ }^{2}$ \\ Marvin E. Adams, ${ }^{1}$ and Stanley C. Froehner ${ }^{1}$
}

${ }^{1}$ Department of Physiology and Biophysics, University of Washington, Seattle. ${ }^{2}$ Cardiovascular Research Center, Massachusetts General Hospital, Boston.

\begin{abstract}
Signaling via the neuronal NOS (nNOS) splice variant nNOS $\mu$ is essential for skeletal muscle health and is commonly reduced in neuromuscular disease. $\mathrm{nNOS} \mu$ is thought to be the predominant source of NO in skeletal muscle. Here we demonstrate the existence of what we believe to be a novel signaling pathway, mediated by the $\mathrm{nNOS}$ splice variant $\mathrm{nNOS} \beta$, localized at the Golgi complex in mouse skeletal muscle cells. In contrast to muscles lacking $\mathrm{nNOS} \mu$ alone, muscles missing both $\mathrm{nNOS} \mu$ and $\mathrm{nNOS} \beta$ were severely myopathic, exhibiting structural defects in the microtubule cytoskeleton, Golgi complex, and mitochondria. Skeletal muscles lacking both $\mathrm{nNOS} \mu$ and $\mathrm{nNOS} \beta$ were smaller in mass, intrinsically weak, highly susceptible to fatigue, and exhibited marked postexercise weakness. Our data indicate that $n N O S \beta$ is a critical regulator of the structural and functional integrity of skeletal muscle and demonstrate the existence of 2 functionally distinct nNOS microdomains in skeletal muscle, created by the differential targeting of nNOS $\mu$ to the sarcolemma and nNOS $\beta$ to the Golgi. We have previously shown that sarcolemmal nNOS $\mu$ matches the blood supply to the metabolic demands of active muscle. We now demonstrate that $\mathrm{nNOS} \beta$ simultaneously modulates the ability of skeletal muscle to maintain force production during and after exercise. We conclude therefore that nNOS splice variants are critical regulators of skeletal muscle exercise performance.
\end{abstract}

\section{Introduction}

Neuronal NOS $\mu$ (nNOS $\mu)$ is a $\mathrm{Ca}^{2+} /$ calmodulin-regulated flavoheme protein that catalyzes the NADPH- and $\mathrm{O}_{2}$-dependent synthesis of the gaseous messenger NO from L-arginine (1). $\mathrm{nNOS} \mu$ is postulated to be the predominant source of $\mathrm{NO}$ in skeletal muscle cells (2). nNOS $\mu$ is present in the cytoplasm and is also localized to the sarcolemma by binding to the scaffold protein $\alpha$-syntrophin, a component of the dystrophin-associated glycoprotein complex $(3,4)$. nNOS catalytic activity is increased several fold by skeletal muscle stimulation, and exercise training increases nNOS $\mu$ expression in both human and rodent muscles $(5,6)$. In most tissues, nNOS signaling is mediated by NO-sensitive soluble guanylyl cyclase (sGC), the principal target of NO. NO stimulates sGC to synthesize cyclic guanosine monophosphate (cGMP), which in turn activates downstream targets, including protein kinase $G(P K G)$ and some ion channels. In skeletal muscle, nNOS $\mu$ performs diverse functions, such as (a) maintaining blood delivery during exercise, (b) modulating glucose homeostasis, (c) controlling muscle mass, and $(d)$ regulating fatigue resistance $(7-10)$. To date, the regulation of blood supply during muscle contraction is the best characterized function of nNOS $\mu$ in skeletal muscle. Contraction-induced production of $\mathrm{NO}$ by sarcolemmal nNOS $\mu$ attenuates $\alpha$-adrenergic receptor-mediated vasoconstriction, thus maintaining appropriate blood and oxygen delivery to active muscles $(7,8)$. Although loss of $\mathrm{nNOS} \mu$ leads to vasoconstriction in contracting muscles (known as functional ischemia), it remains unknown whether this defect

Conflict of interest: The authors have declared that no conflict of interest exists. Citation for this article: J Clin Invest. 2010;120(3):816-826. doi:10.1172/JCI40736. impairs skeletal muscle contractile performance. We recently reported moderate contraction-induced fatigue and reduced postexercise strength in nNOS $\mu$-deficient muscles, suggesting the possibility that functional ischemia could lead to muscle fatigue (10).

$\mathrm{NO}$ was originally thought to function as a freely diffusible messenger, acting on targets distant from its site of synthesis $(11,12)$. This classical view of $\mathrm{NO}$ action raises fundamental questions as to how NO signaling can be selectively directed and targeted to control the many nNOS-regulated pathways described above in skeletal muscle (7-10). It is difficult to envisage this classical mode of action in skeletal muscle due to high concentrations of potent NO scavengers, such as myoglobin or glutathione, that would limit diffusion-based signaling (13). One mechanism postulated to confer specificity to NO signaling is the differential targeting of NOS enzymes with close apposition of effector protein targets, thus facilitating local NO signaling. Evidence for this mechanism has come from studies of nNOS and eNOS signaling in neurons and cardiomyocytes $(12,14,15)$. Support for such a mechanism in skeletal muscle is suggested by the finding that only sarcolemmal $\mathrm{nNOS} \mu$, but not cytoplasmic nNOS $\mu$, can override vasoconstriction in exercising muscle (7). Consequently, we are interested in determining the functions of the sarcolemmal and cytoplasmic nNOS signaling compartments.

Reduced nNOS $\mu$ signaling occurs in many genetically diverse skeletal muscle diseases such as Duchenne muscular dystrophy (DMD), Becker muscular dystrophy, limb-girdle muscular dystrophies (LGMD) 2C, 2D, and 2E, Ullrich congenital muscular dystrophy, and inflammatory myositis $(3,16-18)$. Enhanced NOcGMP signaling improves dystrophic muscle pathology by reduc- 

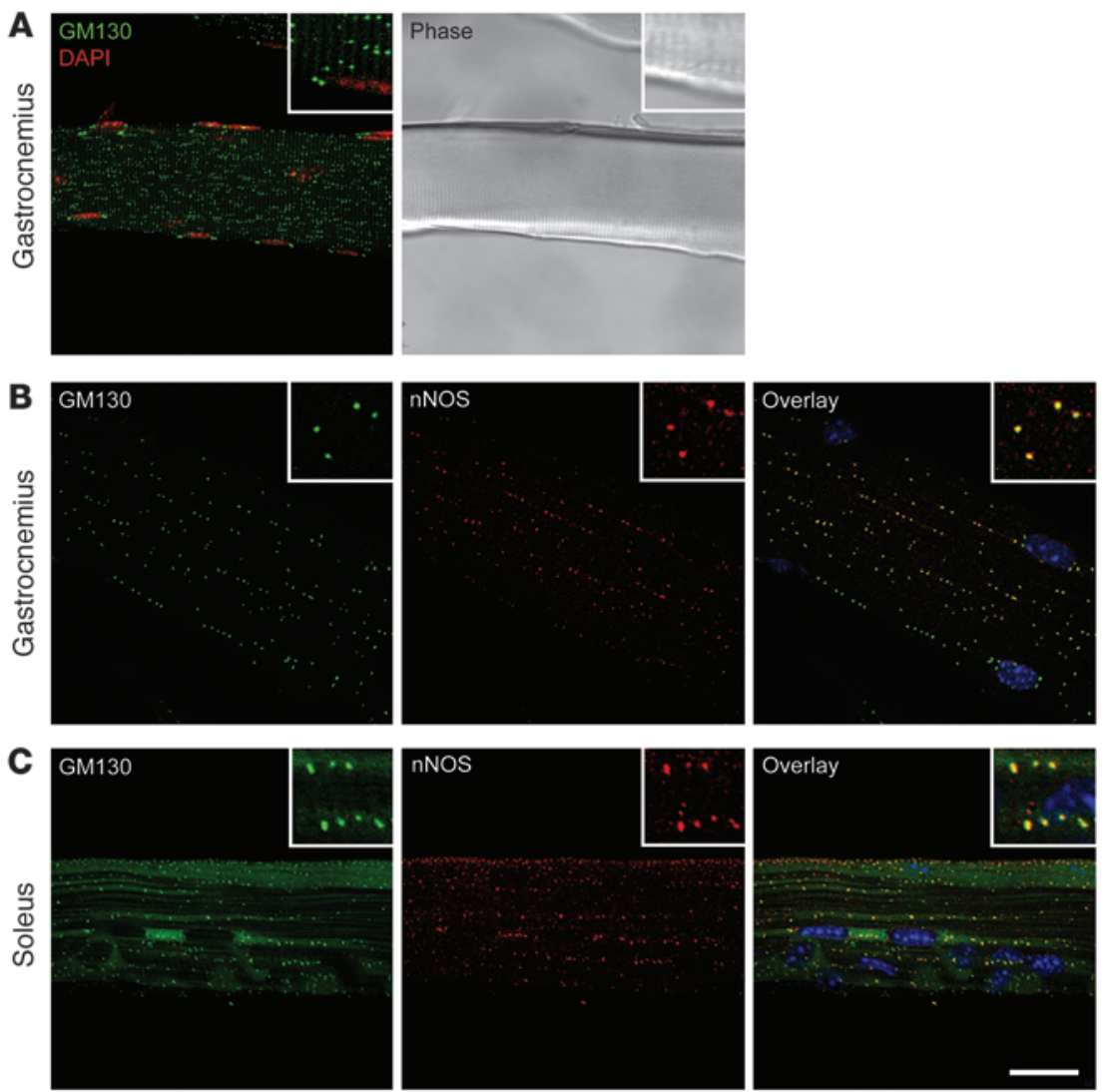

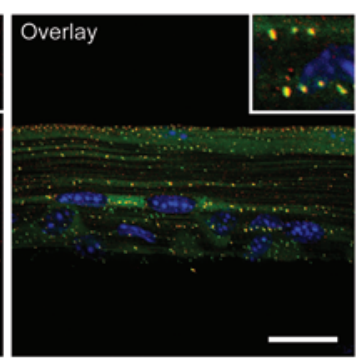

\section{Figure 1}

nNOS is localized to the Golgi complex in fastand slow-twitch skeletal muscle fibers. (A) Golgi complex distribution in fast-twitch gastrocnemius skeletal muscle cells (left panel). The Golgi complex (green) is immunolabeled with a FITC-conjugated GM130 antibody, a marker of cis-Golgi membranes. Myonuclei are counterstained with DAPI (red). A phase contrast image of the fiber is shown in the right panel. (B) nNOS (red) localizes to the Golgi (green) in gastrocnemius myofibers. Surface myonuclei (blue) are labeled with DAPI. The high degree of colocalization is evident in the inset image (yellow) of the overlay panel. (C) nNOS also localizes to the Golgi complex in slowtwitch myofibers of soleus muscle. Higher-magnification images are shown in the inset panels. Scale bar: $20 \mu \mathrm{m} ; 6 \mu \mathrm{m}$ (insets). $n \geq 6$. ing muscle degeneration, decreasing inflammation, and increasing exercise tolerance in mouse models of DMD and LGMD (18-22). Although the precise molecular mechanisms by which $\mathrm{NO}$ ameliorates dystrophy remain to be elucidated, substantial improvements in dystrophic muscle pathology can result from increased cytosolic NO bioavailability and do not require nNOS $\mu$ signaling at the sarcolemma (19). Taken together, these findings further underscore the importance of cytoplasmic NO signaling, particularly in modulating dystrophic pathology, and suggested to us the possibility of additional unrecognized cytosolic NO signaling pathways in skeletal muscle.

In the present study, we set out to (a) define cytoplasmic nNOS signaling pathways in skeletal muscle cells, (b) test the dependence of muscle fatigue on $\alpha$-adrenergic receptor-regulated blood supply during and after exercise, and (c) address whether compartmentalization mechanisms, similar to those observed in cardiomyocytes and neurons, are used to regulate NO signaling specificity in skeletal muscle.

\section{Results}

Approximately half of the nNOS $\mu$ protein expressed in skeletal muscle is bound to the sarcolemmal dystrophin-glycoprotein complex, while the reminder resides in the cytoplasm (1). To determine the location of cytoplasmic nNOS, we immunolabeled single muscle fibers (Figure 1A), using a method optimized for the resolution of subcellular organelles (23). Four pan-specific anti-nNOS antibodies raised against distinct epitopes detected nNOS localized to sub-sarcolemmal puncta (Figure 1, B and C, and Supplemental Figure 1; supplemental material available online with this article; doi:10.1172/JCI40736DS1). Sarcolemmal nNOS $\mu$ labeling is present in this fiber but is out of the plane of focus. The punctate labeling pattern resembled that of the murine skeletal muscle Golgi complex (23). In fact, nNOS puncta colocalized precisely with GM130, a marker of the cis-face of the Golgi complex in fast-twitch gastrocnemius (Figure 1B) and slow-twitch soleus muscle fibers (Figure 1C). These data suggest the existence of a previously unrecognized Golgi-associated nNOS compartment in skeletal muscle cells.

To establish the identity of the Golgi nNOS, we used nNOS1 knockout 1 (KN1, knockout of nNOS $\mu$ only; Figure 2D) and nNOS knockout 2 (KN2, knockout of any active full-length nNOS splice variants; Figure 2E) mice (see Methods and refs. 24, 25). At least 4 splice variants of nNOS may be transcribed from the Nos 1 gene; however, $\mathrm{nNOS} \alpha$ is not expressed in skeletal muscle, leaving only nNOS $\mu, \mathrm{nNOS} \beta$, and nNOS $\gamma$ as possible candidates for Golgi nNOS (Figure 2A). Surprisingly, Golgi nNOS labeling was unaffected in KN1 skeletal muscles (Figure 3A), indicating that the Golgi nNOS was not nNOS $\mu$. Golgi nNOS labeling was absent in skeletal muscles from KN2 mice, further confirming nNOS antibody specificity (Figure 3A). Taken together, these data suggest that the Golgi-associated nNOS is either nNOS $\beta$ and/or nNOS $\gamma$. While we cannot definitively eliminate nNOS $\gamma$ as a candidate for the Golgi nNOS, it seems unlikely. Unlike nNOS $\beta$, which is catalytically normal and possesses a unique amino terminus, $\mathrm{nNOS} \gamma$ is enzymatically inactive and lacks unique primary sequence to facilitate Golgi targeting (Figure 2A) (4). Therefore, throughout this paper, we will refer to skeletal muscle Golgi nNOS as nNOS $\beta$. More importantly, these data suggest the existence of 3 distinct 
A PDZ Heme $\quad$ Herinsert

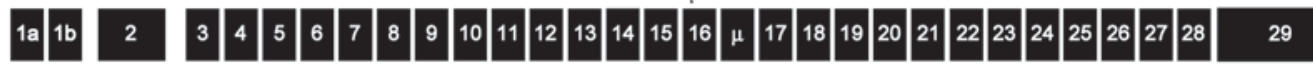
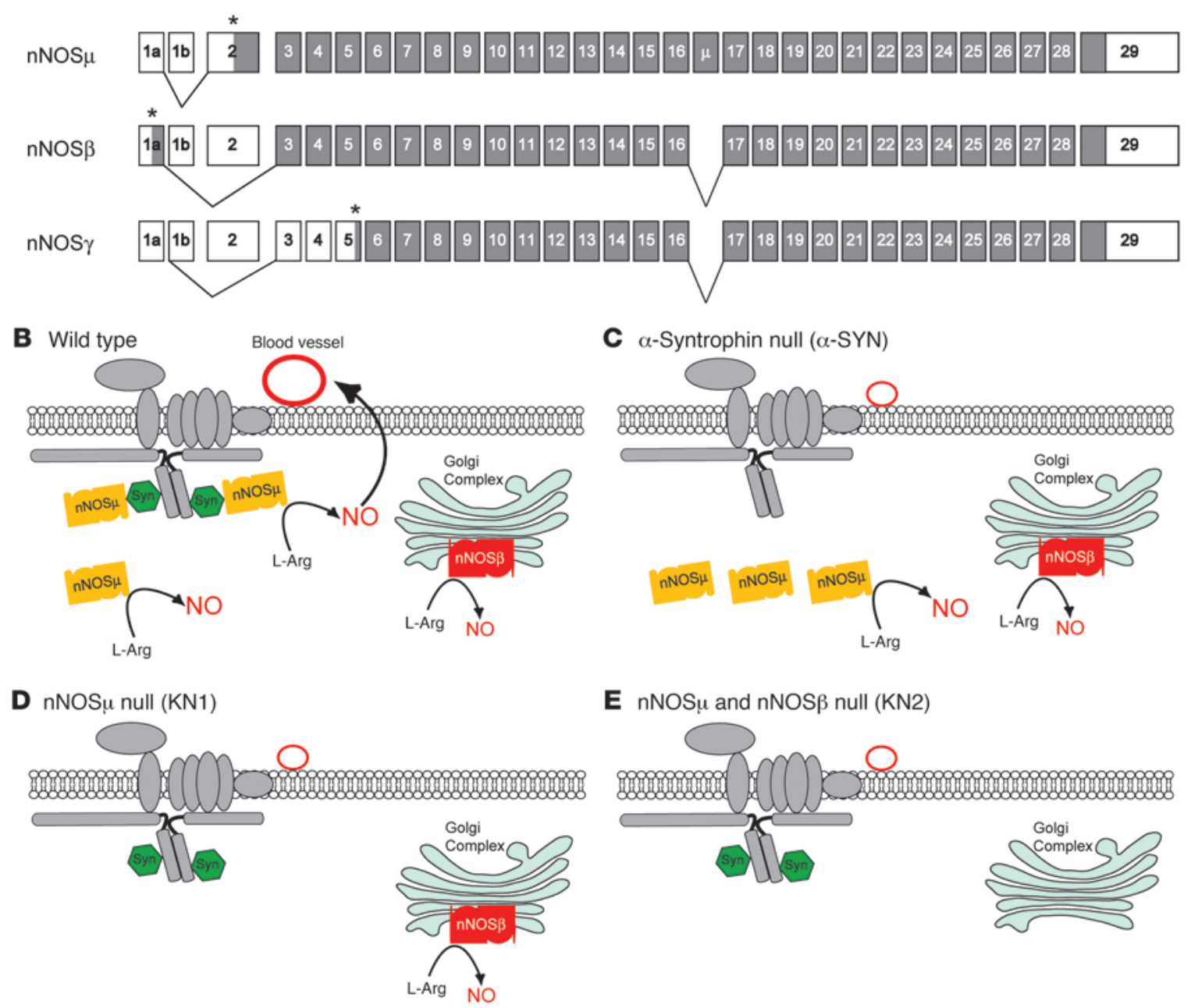

E nNOS $\mu$ and $\mathrm{nNOS} \beta$ null (KN2)

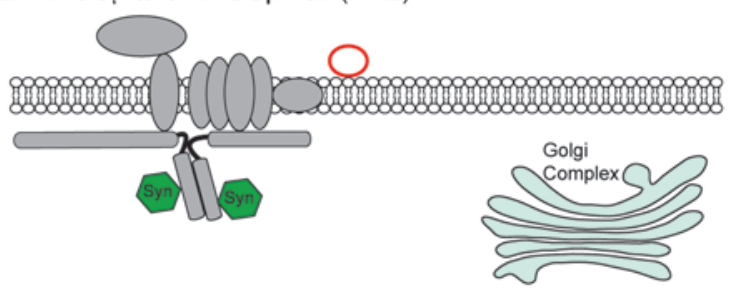

Figure 2

nNOS splice variants and nNOS mutant mouse lines used to analyze NO signaling in skeletal muscle. (A) Exon structure of the murine Nos1 gene, which encodes 31 exons (black boxes) (39). Relevant isozymes are shown here. The coding sequence is shown in gray, and asterisks mark translation initiation sites. The 5'-and 3'-untranslated sequences are shown as white boxes. In skeletal muscle, the predominant transcript is nNOS $\mu$ containing exon 2 (encoding the PSD-95, discs-large, ZO-1 [PDZ] domain) and the 34-amino acid $\mu$-insert. nNOS $\beta$ is formed by the splicing of exon 1 a to exon 3 , creating a unique 6 -amino acid N terminus. nNOS $\gamma$ is produced by translation initiation at an internal ATG site in exon 5. (B) WT muscle can express all nNOS splice variants. nNOS $\mu$ (yellow) localizes to the sarcolemma by binding $\alpha$-syntrophin (Syn; green), a member of the dystrophin-glycoprotein complex (gray). nNOS $\mu$ also localizes to the cytoplasm. In contracting muscles, sarcolemmal nNOS $\mu$ derived NO overrides sympathetic vasoconstriction maintaining blood vessel dilation (large black arrow) (7). In the present study, we identify a splice variant of nNOS localized to the Golgi complex, presumed to be nNOS $\beta$ (red). The small black arrows represent the synthesis of NO from L-arginine by nNOS. (C) Muscles lacking $\alpha$-syntrophin cannot localize nNOS $\mu$ to the sarcolemma and thus cannot attenuate vasoconstriction, despite expression of cytosolic nNOS $\mu$ and Golgi nNOS $\beta$. (D) The muscles of KN1 mice do not express nNOS $\mu$, due to deletion of exon 2, but $n N O S \beta$ and nNOS $\gamma$ splice variant production is unaffected (24). (E) KN2 mice do not express any full-length active nNOS splice variants due to deletion of exon 6 encoding the heme-binding domain (25).

nNOS compartments (Golgi nNOS $\beta$, sarcolemmal nNOS $\mu$, and cytoplasmic nNOS $\mu$ ) and reveal the previously unrecognized complexity of nNOS-based signaling in skeletal muscle.

Classical NO signal transduction is mediated by NO-sensitive sGC and PKG. To test the possibility that NO-cGMP signaling could occur at the skeletal muscle Golgi complex, we examined whether sGC and PKG localized to Golgi membranes. Both sGC (Figure 4A) and PKG (Figure 4B) colocalized with GM130. These data suggest that sGC and PKG, the primary targets and effectors of NO, are in close proximity to nNOS $\beta$ at the Golgi. These data strongly support the possible existence of a novel nNOScGMP signal transduction microdomain at the Golgi complex in skeletal muscle.

Skeletal muscles from KN1 and KN2 mice were examined to determine the subcellular consequences of nNOS splice variant deficiency on skeletal muscle organization. The absence of nNOS 
A
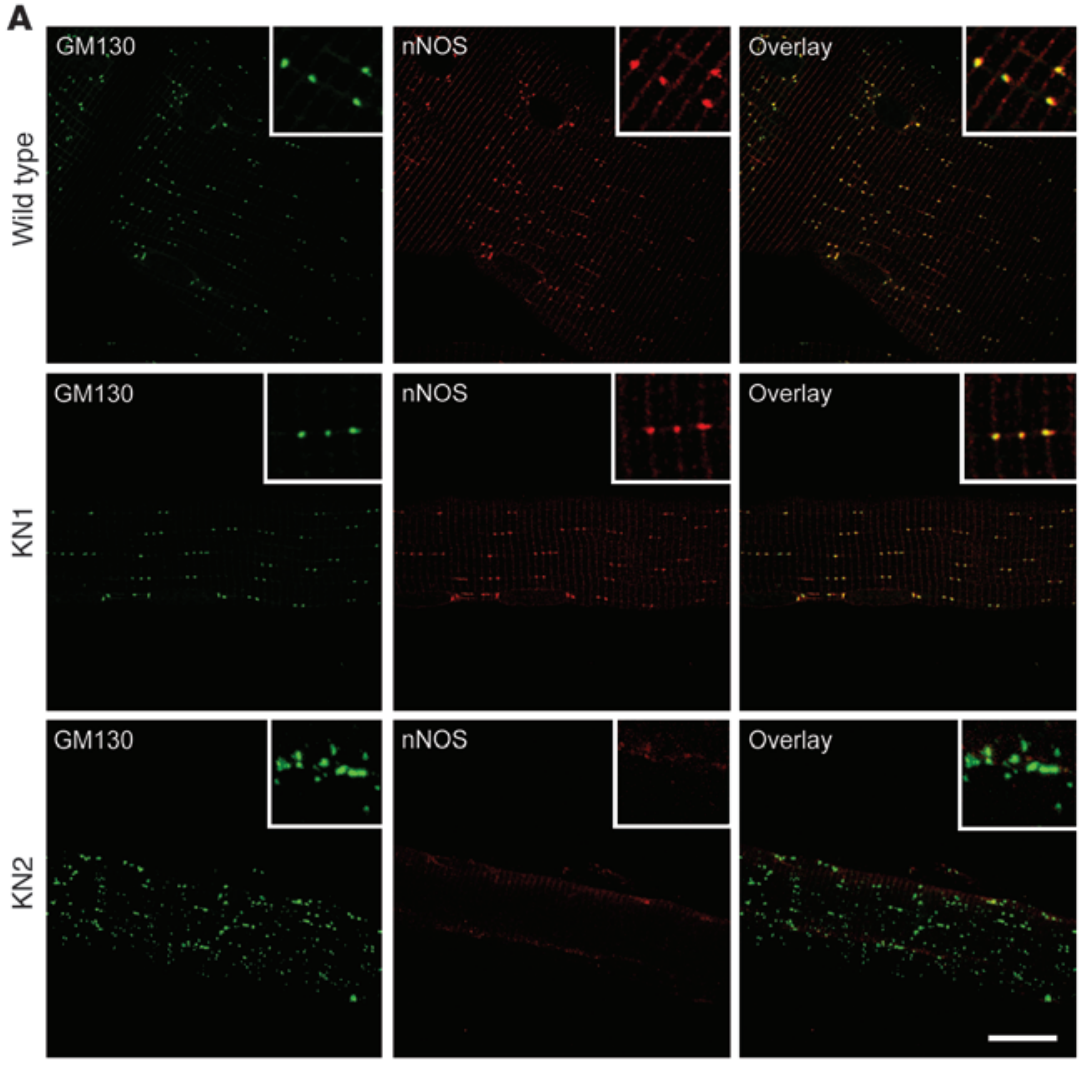

B
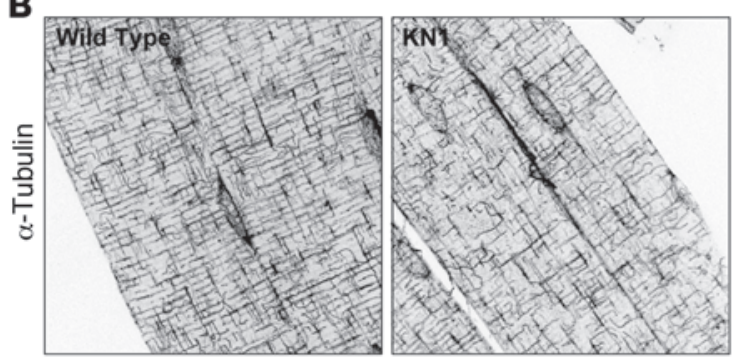

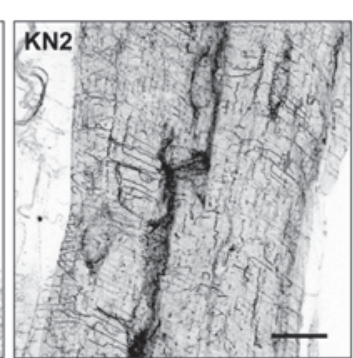

\section{Figure 3}

A nNOS splice variant localizes to the Golgi complex and regulates microtubule cytoskeleton integrity in skeletal muscle. (A) In WT gastrocnemius muscles, nNOS (red) colocalizes with GM130 (green). The inset overlay image emphasizes the high degree of overlap between GM130 and Golgi nNOS. In KN1 muscles, Golgi nNOS labeling is unaffected, indicating that the Golgi nNOS is not nNOS $\mu$ but nNOS $\beta$ and/or nNOS $\gamma$. Golgi nNOS immunolabeling is absent in KN2 muscles, demonstrating nNOS antibody specificity. The loss of all nNOS variants perturbs the distribution and morphology of the Golgi complex. Higher-magnification images are shown in the inset panels. (B) nNOS splice variants differentially regulate the structural integrity of the microtubule cytoskeleton. Microtubules were labeled with FITC-conjugated $\alpha$-tubulin antibody. The characteristic microtubule lattice is evident in WT myofibers (left panel). Loss of nNOS $\mu$ has a minor impact on microtubule organization (middle panel), which was insufficient to disrupt Golgi distribution. The loss of all nNOS splice variants severely compromises the integrity of the microtubule cytoskeleton (right panel). Scale bar: $20 \mu \mathrm{m} ; 6 \mu \mathrm{m}$ (insets). $n \geq 4$. in the muscles of KN2 mice disrupted the distribution of the Golgi (Figure 3A, bottom row), which was unaffected in $\mathrm{KN} 1$ relative to WT muscle (Figure 3A, middle and top rows). Since microtubules are responsible for Golgi complex localization (26), we evaluated microtubule cytoskeleton organization in nNOS mutant mice (Figure 3B). The orthogonal lattice of microtubules was unaffected by the absence of nNOS $\mu$ (Figure 3B). In contrast, the microtubule cytoskeleton was dramatically disrupted in nNOS-deficient muscles of KN2 mice (Figure 3B). These data suggest that nNOS $\beta$ regulates microtubule cytoskeleton integrity. nNOS $\beta$ deficiency contributes to destabilization of the microtubule lattice and perturbation of Golgi membrane organization.

Muscle fatigue resistance is an excellent global measure of muscle performance. We evaluated this parameter in tibialis anterior (TA) muscles in situ from KN1, KN2, and $\alpha$-syntrophin-null mice (Figure 2C and Figure 5 and Table 1). nNOS $\mu$ fails to localize to the sarcolemma in $\alpha$-syntrophin-null muscle, despite preservation of nNOS $\mu$ expression and activity; therefore $\alpha$-syntrophin knockouts are especially useful in specifically figuring out sarcolemmal $\mathrm{nNOS} \mu$ function $(7,27)$. Given the importance of contraction- induced nNOS $\mu$ signaling in vascular function, we used an in situ method for testing muscle contractile function, in which normal vascularization and innervation of skeletal muscle tissue are maintained. After 4 minutes of simulated exercise, force output from WT TA muscles was 50\% of initial levels (Figure 5 and Table 1). The loss of sarcolemmal nNOS $\mu$ only in the $\alpha$-syntrophin knockout (Figure 2C) had no significant impact on contraction-induced fatigue or postexercise force generation despite aberrant vasoconstriction (Figure 5 and Table 1) (7). Contrary to expectation, the absence of muscle nNOS $\mu$ in KN1 mice also had no impact on muscle fatigue or postexercise strength (Figure 5 and Table 1). Using an identical approach, we previously reported mild contraction-induced fatigue in nNOS $\mu$-deficient TA muscles in KN1 mice on a B6129 background $(10,24)$. The mice in this study were all generated on, or backcrossed 10 generations onto, a C57BL/6 background. These data demonstrate strain-specific modulation of nNOS $\mu$-regulated fatigue. Our data also argue that the functional ischemia in the absence of contraction-induced sarcolemmal nNOS $\mu$ signaling does not affect susceptibility to contractioninduced fatigue or postexercise force generating capacity. 

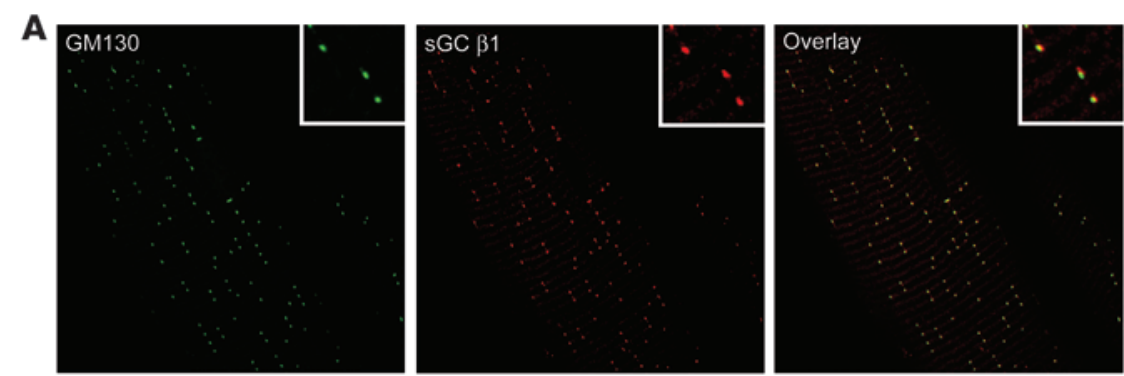

B $\mathrm{GM} 130$
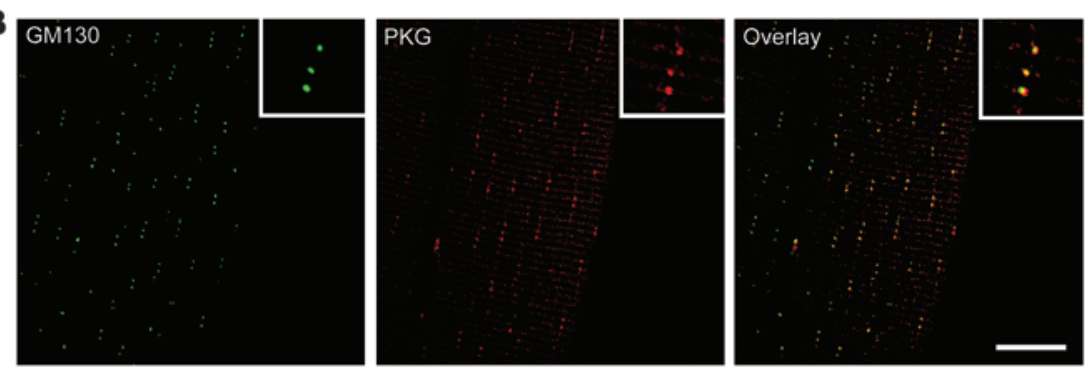

\section{Figure 4}

A NO-cGMP signaling microdomain that we believe to be novel at the Golgi complex in skeletal muscle. (A) Gastrocnemius myofibers were coincubated with antibodies against the cisGolgi marker GM130 and the NO-binding $\beta 1$ subunit of sGC (sGC $\beta 1$ ). GM130 and the NObinding $\beta 1$ subunit of sGC showed a high degree of overlap in gastrocnemius muscle cells (right panel). (B) Isolated myofibers were separately colabeled with antibodies against GM130 and PKG. GM130 and PKG showed a high degree of overlap in skeletal muscle cells (right panel). These data demonstrate that compartmentalization of nNOS and effector proteins occurs at the Golgi complex in skeletal muscle. Higher-magnification images are shown in the inset panels. Scale bar: $20 \mu \mathrm{m} ; 6 \mu \mathrm{m}$ (insets). $n \geq 6$.
In contrast, the loss of all nNOS splice variants in KN2 muscle dramatically increased susceptibility to contraction-induced fatigue (Figure 5 and Table 1). KN2 skeletal muscles also exhibited significant force deficits after exercise. Postexercise weakness was apparent in KN2 muscles even at 5 minutes after exercise (Figure 5 and Table 1). These data provide 4 insights, which we believe to be new, into nNOS splice variant function: (a) loss of contraction-induced signaling from nNOS $\mu$ does not necessarily limit force production during or after exercise; (b) nNOS $\beta$ is a critical regulator of skeletal muscle fatigue and postexercise force output; (c) during exercise, nNOS $\mu$ signaling maintains blood delivery to active muscle, while $\mathrm{nNOS} \beta$ regulates muscle fatigue resistance and postexercise force output; and (d) the differential targeting of nNOS splice variants creates functionally distinct NO signaling microdomains, at which NOS-derived NO acts locally in skeletal muscle.

A possible molecular explanation for increased muscle fatigue in $\mathrm{KN} 2$ muscle is a decrease in the ratio of fatigue-resistant to fatiguesusceptible muscle fibers. To address the possibility that altered fiber composition contributes to increased KN2 muscle fatigue, we determined the frequency of fast-and slow-twitch fibers in nNOS mutant TA muscles (Figure 6A). Fibers expressing type I myosin heavy chain $(\mathrm{MyHC})$ are the most fatigue resistant, while those expressing type IIb MyHC are the least fatigue resistant (28). Type IIapositive myofibers exhibit an intermediate fatigue resistance. Type IIx/IId fibers are also relatively resistant to fatigue (28). KN1 muscles exhibited a shift to a more fatigue-resistant fiber composition, characterized by a significant $50 \%$ reduction in type IIa-positive fibers and remarkable 3,000\% increase in type IIx/IId fibers compared with normal controls (Figure 6B and Supplemental Figure 2). The marked increase in type IIx/IId fibers could contribute to the fatigue resistance of nNOS $\mu$-deficient KN1 TA muscle. Faster mean relaxation and peak twitch force generation times are also consistent with the increased type IIx/IId fast fiber composition of KN1 TA muscle (Supplemental Figure 3). In contrast, there was a significant $45 \%$ increase relative to WT in type IIb fibers in nNOSdeficient KN2 muscles that could contribute to decreased fatigue resistance. The shift toward more fatigue-resistant and fatiguesusceptible fiber compositions in KN1 and KN2 muscles, respec- tively, provides a potential mechanism for the differential impact of nNOS splice variants on contraction-induced muscle fatigue.

In order to determine the full extent of nNOS splice variant regulation of skeletal muscle contractile function, we determined maximal tetanic force generating capacity and specific force (total tetanic force normalized to muscle cross-sectional area [CSA]) for all nNOS genotypes. Maximum tetanic force output did not differ significantly between WT, $\alpha$-syntrophin-deficient and KN1 TA muscles (Figure 7A). In contrast, KN2 muscles lacking nNOS $\mu$ and $\mathrm{nNOS} \beta$ generated only $40 \%$ of the maximum isometric force of WT controls (Figure 7A). Similarly, KN2 muscles were significantly weaker, exhibiting a $14 \%$ reduction in mean specific force (Figure 7B). Thus, nNOS $\beta$-derived NO appears necessary to maintain normal skeletal muscle strength.

Since muscle strength correlates positively with muscle size, we investigated whether the intrinsic weakness of KN2 muscle was due to a reduction in muscle mass. The mass of $\alpha$-syntrophin-null and KN1 TA muscles did not differ from controls (Figure 7C); however KN2 muscle mass decreased 40\% $(P<0.001)$ compared with WT controls. Normalization of muscle mass to body weight demonstrated that the decrease was not simply attributable to decreased body mass (data not shown). The median CSA of KN2 TA myofibers was significantly smaller $\left(1,382 \mu \mathrm{m}^{2}\right)$ than that of controls $\left(1,773 \mu \mathrm{m}^{2}\right)$ and KN1 muscle cells $\left(1,814 \mu \mathrm{m}^{2}\right.$; Figure 7D). Thus, decreased muscle mass in KN2 mice is likely due to a reduction in the size of the muscle cells themselves.

We then tested whether all fiber types were equally reduced in size by the absence of nNOS $\beta$ (Supplemental Figure 2). We measured the CSA of type IIa and type IIb fibers, which together make up approximately $90 \%$ of the myofibers in WT and KN2 TA muscles. The CSA of type IIb fibers was significantly decreased by $31 \%$ $(P<0.05)$ in KN2 TA muscles, while the area of type IIa fibers was not significantly affected (Supplemental Figure 2B). It is important to note the $45 \%$ increase in the number of type IIb fibers per square millimeter in KN2 TA muscles (Figure 6 and Supplemental Figure 2); therefore, the decrease in KN2 TA muscle mass is not simply due to a change in fiber type but is attributable to, at least in part, increased numbers of significantly smaller type IIb fibers. 


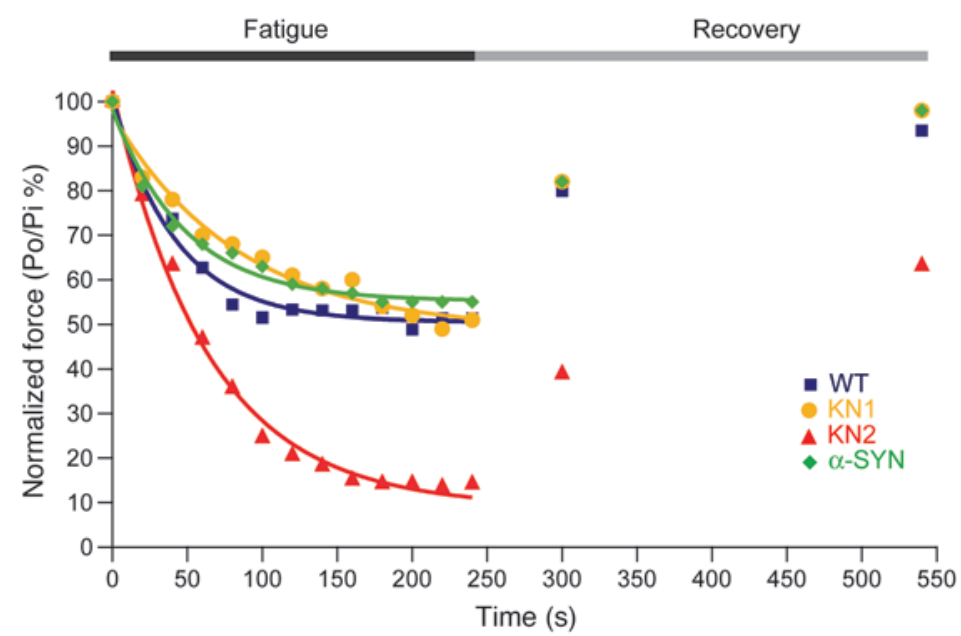

Figure 5

nNOS splice variants differentially regulate contraction-induced fatigue and postexercise force recovery. TA muscles were subjected to a series of maximal tetanic stimulations every 2 seconds for 4 minutes to simulate exercise. Muscles were rested, then maximally stimulated once at 1 and 5 minutes to measure postexercise strength recovery. Representative traces for each nNOS mutant fitted with exponential decay curves are shown. TA muscles of $\alpha$-syntrophin ( $\alpha$-SYN) knockout mice (green diamonds) or KN1 muscles (yellow circles) exhibit normal muscle fatigue indistinguishable from WT controls (blue squares). In contrast, KN2 muscles (red triangles) exhibited significantly increased susceptibility to contraction-induced muscle fatigue, with force output levels leveling off at approximately $60 \%$ lower than those from WT, KN1, and $\alpha$-syntrophin muscles. Force recovery at 1 and 5 minutes was significantly decreased by approximately $50 \%$ after simulated exercise, indicating substantial postexercise weakness. Po is the tetanic force in millinewtons generated at time $t$ during the stimulation protocol. $\mathrm{Pi}$ is the initial force output in millinewtons at time 0 , at the beginning of the experiment.

In contrast, $\mathrm{nNOS} \mu$ deficiency resulted in a significant decrease in KN1 soleus muscle mass and median CSA relative to WT (Supplemental Figure 4). However, similar to the TA, KN2 soleus muscles were also smaller in mass and CSA (Supplemental Figure 4). These data suggest that $\mathrm{nNOS} \beta$ signaling pathways regulate muscle mass and myofiber size independently of muscle type, whereas nNOS $\mu$ signaling does so through muscle type-specific mechanisms.

Destabilization of the microtubule cytoskeleton, mislocalization of the Golgi complex, and reduced myofiber size were obvious myopathic changes evident from light microscopy-based analyses of WT, KN1, and KN2 muscles (Figure 8A and Supplemental Figure 4). To identify additional myopathic changes in muscle cytoarchitecture, we examined skeletal muscles at the ultrastructural level. Electron microscopic analysis of KN1 TA muscle revealed intermyofibrillar mitochondria that were swollen and electron lucent (presumably reflecting decreased matrix density) (Figure 8B). Sarcomere organization and registration in $\mathrm{KN} 1$ muscle were indistinguishable from that of controls (Figure 8B). In KN2 TA muscles, intermyofibrillar mitochondria were often more swollen and variable in size, electron lucent, and aberrantly localized (Figure 8B). This is consistent with defects in the organization of the microtubule cytoskeleton that anchors mitochondrial organ- elles (Figure 3B) (29). Furthermore, regions of muscle appeared highly disorganized, with impaired sarcomere alignment. To test whether structural abnormalities in nNOS-deficient mice lead to increased muscle fragility, thus predisposing TA muscles to contraction-induced injury, force output was determined at progressively increasing strains. $\alpha$-Syntrophin-null, KN1, and KN2 muscles exhibited force deficits that were indistinguishable from that of WT controls (Supplemental Figure 5). Similarly, creatine kinase activity was unaffected in the serum from KN1 (ref. 18 and data not shown) and KN2 (Supplemental Figure 6) mice, indicating normal myofiber stability and turnover. Together, these data show that nNOS splice variant deficiency does not increase susceptibility to contractioninduced injury or decrease muscle cell stability, in agreement with a previous study (10), and do suggest an important role for both nNOS $\mu$ and $n N O S \beta$ splice variants in maintaining normal mitochondrial health.

\section{Discussion}

The principal finding of this study is the identification of what we believe to be a novel nNOS $\beta$ signaling transduction pathway at the Golgi complex, revealing previously unrecognized NO signaling complexity in skeletal muscle. Skeletal muscle contains at least 3 spatially and functionally distinct nNOS signaling microdomains: (a) the sarcolemmal nNOS $\mu$ compartment, (b) the cytoplasmic nNOS $\mu$ compartment (whose function remains to be determined), and (c) the Golgi nNOS compartment composed of nNOS $\beta$ and its likely downstream effectors sGC and PKG (Figure 9). In our working model of nNOS signaling in exercising muscle (Figure 9), excitation-contraction coupling leads to increased intracellular $\mathrm{Ca}^{2+}$ levels, allowing $\mathrm{Ca}^{2+} /$ calmodulin to bind and activate nNOS $\mu$ and nNOS $\beta$. nNOS $\mu$ attenuates local $\alpha$-adrenergic receptor-mediated vasoconstriction, thereby maintaining appropriate blood and oxygen supply to active muscle tissue $(7,8)$, while nNOS $\beta$ simultaneously functions to maintain contractile force output during exercise and postexercise force generation (Figure 9). NO may regulate contractility by cGMP-dependent activation of PKG and other cGMP targets, such as ion channels, or by cGMP-independent signaling mechanisms, such as nitrosylation of target substrates (Figure 9).

$\mathrm{nNOS} \mu$ and nNOS $\beta$ splice variants differentially regulate skeletal muscle strength, susceptibility to contraction-induced fatigue, and postexercise force output. In the $\mathrm{C} 57 \mathrm{BL} / 6$ background used

\section{Table 1}

Skeletal muscle fatigue in KN1, KN2, and $\alpha$-syntrophin-null mice

\begin{tabular}{lcccc}
\hline & & & & \\
& WT & \multicolumn{1}{c}{ KN1 } & \multicolumn{1}{c}{ KN2 } & $\alpha$-Syntrophin \\
Force plateau (\%) & $52.7 \pm 3.6$ & $47.4 \pm 3.7$ & $21.9 \pm 4.9^{A, B, C}$ & $56.0 \pm 1.8$ \\
Time constant (s) & $38.1 \pm 4.3$ & $60.2 \pm 9.0$ & $53.9 \pm 4.6$ & $53.2 \pm 5.3$ \\
Recovery (1 min) (\%) & $81.2 \pm 2.5$ & $77.0 \pm 2.1$ & $48.5 \pm 7.0^{A, B, D}$ & $79.7 \pm 1.4$ \\
Recovery (5 min) (\%) & $95.2 \pm 1.8$ & $90.1 \pm 2.9$ & $64.5 \pm 7.3^{\mathrm{A}, \mathrm{D}, \mathrm{E}}$ & $91.3 \pm 1.7$
\end{tabular}

Values (mean \pm SEM) are shown for the force plateau (asymptote), time constant, and postexercise force recovery at 1 and 5 minutes after exercise. Mean time constant values were unaffected. KN1 and $\alpha$-syntrophin mice also exhibited normal postexercise force recovery at 1 and 5 minutes. ${ }^{A} P<0.001$, WT versus $\mathrm{KN} 2$. ${ }^{\mathrm{B} P}<0.01$, $\mathrm{KN} 1$ versus $\mathrm{KN} 2 .{ }^{C} P<0.001$, $\mathrm{D} P<0.01, \alpha$-syntrophin versus KN2. $E P<0.05, \mathrm{KN} 1$ versus KN2. $n \geq 6$ for each group. 

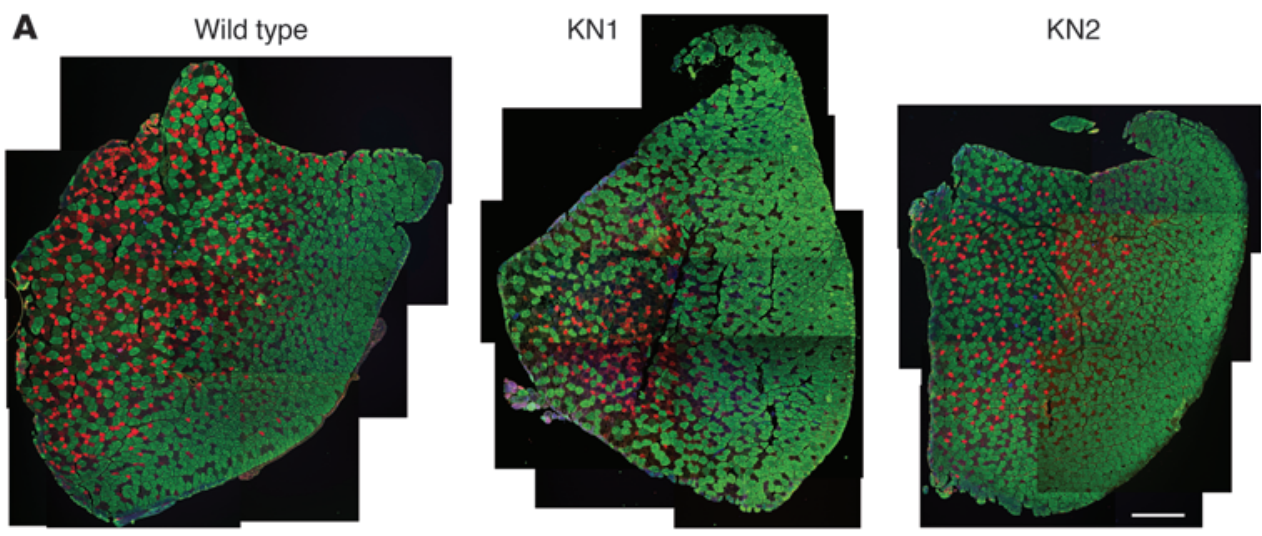

is that absence of $\mathrm{nNOS} \beta$ signaling may decrease muscle mass, in part through downregulation of follistatin growth factor expression (30). Taken together, these data provide a compelling case for a crucial role of nNOS $\beta$ in regulating muscle mass, muscle strength, muscle fatigue, and postexercise force output.

Muscle nNOS splice variants also differentially impact the structural properties of skeletal muscle. One striking example is the disruption of the microtubule cytoskeleton observed in nNOSdeficient KN2 muscle, which suggests a role for Golgi nNOS $\beta$ in regulating the stability of the microtubule polymer system. $\alpha$-Tubulin is nitrosylated in vivo; therefore, loss of nNOS could lead to tubulin hyponitrosylation and polymer instability (31). Given the well-described function of microtubules in positioning of subcellular organelles $(26,29)$, microtubule cytoskeleton destabilization may cause the aberrant localization of the Golgi complex and intermyofibrillar mitochondria in KN2 muscle. Parallel disruption of microtubules and Golgi complex distribution has also been reported in dystrophic myofibers (23). Interestingly, mitochondria were also structurally compromised in both KN1 and KN2 muscles, suggesting a mitochondrion protective role for nNOS isozymes (32).

Mitochondrial abnormalities may

in this study, nNOSu does not regulate muscle fatigue, suggesting that the functional ischemia resulting from aberrant $\alpha$-adrenergic receptor-mediated vasoconstriction does not impact the ability of muscle to generate force during or after exercise. However, muscles lacking both nNOS $\mu$ and nNOS $\beta$ exhibited profound muscle fatigue and postexercise weakness. These data strongly suggest that $\mathrm{nNOS} \beta$ plays a major role in muscle fatigue and postexercise force generation. Similarly, nNOS $\beta$ but not nNOS $\mu$ is required to maintain normal muscle strength.

Deficits in total force-generating capacity in nNOS-deficient muscles were likely attributable in part to reductions in muscle mass. Decreased muscle mass in KN2 mice was at least partly attributable to increased numbers of abnormally small type IIb fibers, suggesting the inability of type IIb fibers to hypertrophy to the correct size in the absence of nNOS $\beta$. These data suggest that decreased KN2 muscle mass, and its characteristic susceptibility to fatigue, are the result of fiber-specific changes resulting from nNOS $\beta$ deficiency. Other nNOS-mediated mechanisms regulating muscle mass may also be involved. An intriguing possibility also be a substantial contributing factor to contraction-induced fatigue in $\mathrm{KN} 2$ mice. These data suggest that nNOS-derived NO is essential for normal mitochondrial health and for microtubule cytoskeleton integrity.

These findings highlight the existence of functionally distinct, parallel nNOS signaling pathways and provide fresh insight into how independent signaling function may be achieved in skeletal muscle. NO is classically described to function as a promiscuous and highly diffusible messenger, capable of crossing membranes to adjacent cells over distances of $100 \mu \mathrm{m}$ or more (11). It is difficult to envision the existence of such a nonspecific mechanism, given the high concentrations of myoglobin in skeletal muscle that can rapidly scavenge NO (13). Furthermore, the redundancy in NO signaling predicted by this classical view is not support by the data in the current or previous muscle studies. First, NO synthesized by cytoplasmic nNOS $\mu$ cannot compensate for the absence of sarcolemmal nNOS $\mu$ and prevent vasoconstriction during exercise in $\alpha$-syntrophin-null muscle (7). Second, despite its localization near the sarcolemma, Golgi nNOS $\beta$ expression also cannot 
A
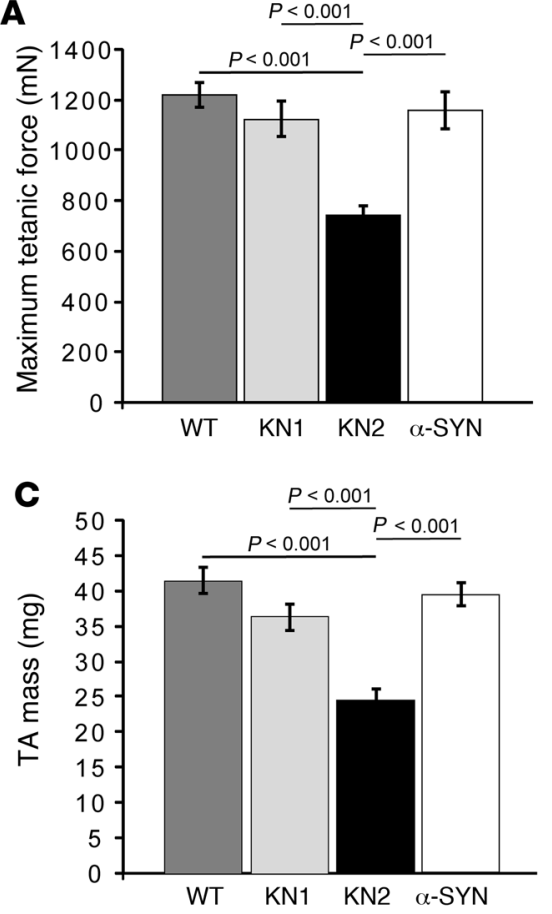

B

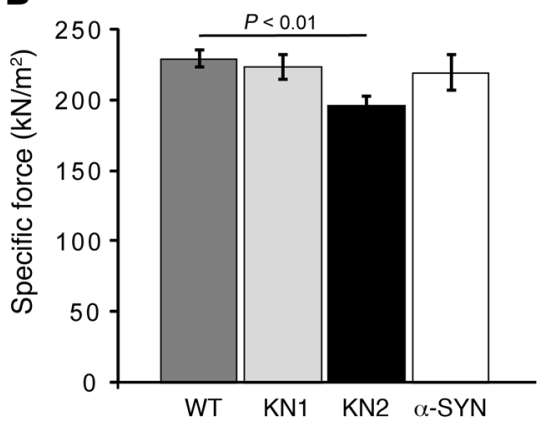

D

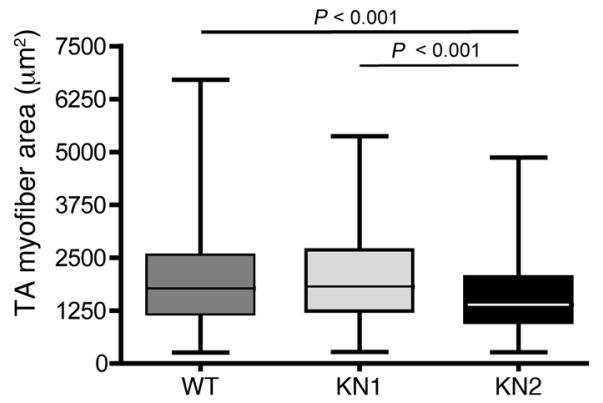

Figure 7

Golgi nNOS splice variant is required to maintain normal skeletal muscle strength, muscle mass, and myofiber size. (A) Maximum isometric force-producing capacity of TA muscles from KN1 and $\alpha$-syntrophin mice did not differ significantly from WT controls. nNOS-deficient KN2 muscles exhibited significantly reduced maximum force-generating capacity $(P<0.001)$ compared with controls, KN1, and $\alpha$-syntrophin mice. (B) Mean specific force did not differ statistically between WT controls, KN1, and $\alpha$-syntrophin mice. The additional loss of $\mathrm{nNOS} \beta$ in KN2 mice lead to a significant $14 \%$ decrease $(P<0.01)$ in specific force, indicating that $n N O S \beta$ is required for normal muscle strength. (C) TA muscle weight was unaffected in $\alpha$-syntrophin mice and KN1 mice; however, loss of nNOS $\mu$ and nNOS $\beta$ lead to a significant $40 \%$ reduction $(P<0.001)$ in muscle mass. (D) The CSA of KN1 myofibers was similar to WT controls. KN2 muscle cells were significantly smaller in area $(P<0.001)$, suggesting that decreased myofiber size could at least partially account for decreased KN2 muscle mass. Values represent mean \pm SEM $(\mathbf{A}, \mathbf{B}$, and $\mathbf{C})$. The median is represented by the line within the box plot in $\mathbf{D}$. Group sizes in $\mathbf{A}, \mathbf{B}, \mathbf{C}$, and $\mathbf{D}$ are $n \geq 6, n \geq 6, n \geq 9$, and $n \geq 4$, respectively.

prevent vasoconstriction during contraction in nNOS $\mu$-deficient muscles from KN1 mice $(7,8)$. Third, because of the theoretical diffusibility of Golgi nNOS-derived NO, the classical view predicts that the phenotypes of KN1 muscles should be virtually indistinguishable from WT controls. Instead, nNOSu-deficient muscles differ from WT muscle in at least 4 respects: they exhibit aberrant local vasoconstriction during exercise $(7,8)$, reduced soleus muscle mass, unique fiber type composition, and morphologically aberrant mitochondria. It is important to note that the possibility of partial redundancy between NO signaling pathways cannot be completely ruled out; however, this is a separate issue from the free diffusibility of NO. Nevertheless, the differential targeting of $\mathrm{nNOS} \mu$ and nNOS $\beta$ to create 2 spatially and functionally distinct NO signaling microdomains suggests an important mechanism for regulating NO signaling specificity. These findings provide a compelling argument that nNOS splice variant-derived $\mathrm{NO}$ is produced and acts locally in skeletal muscle.

Our results are consistent with previous studies of NOS signaling that highlight the importance of NOS enzyme subcellular local- of target proteins by nNOS, and (c) by reacting with $\mathrm{NO}$ to generate cytotoxic peroxynitrite. Conversely, transgenic or pharmacologic approaches that ameliorate the dystrophic phenotype of mouse models of muscular dystrophy by increasing NO bioavailability or NO-cGMP signaling may have serendipitously activated Golgi-localized NO-activated sGC, leading to increased levels of cGMP second messengers (18-21). Thus the beneficial effects of increased cytoplasmic NO in dystrophic muscle may be due, at least in part, to activation of Golgi NOcGMP signaling pathways. Regardless, and perhaps more importantly, the Golgi nNOS $\beta$ signaling pathway provides a potential new therapeutic target for treating neuromuscular disease, including muscular dystrophy.

How might dysregulation of nNOS $\mu$ signaling contribute to dystrophic pathology? Loss of contraction-induced sarcolemmal nNOS $\mu$ signaling leads to functional ischemia $(7,8,34)$. Although this is associated with a modest increase in fatigue in KN1 mice on a B6/129 genetic background (10), our data on mice in a C57BL/6 background do not support a causal relationship between functional ischemia and muscle fatigue or postexercise strength. 


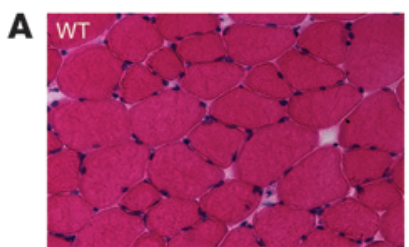

B
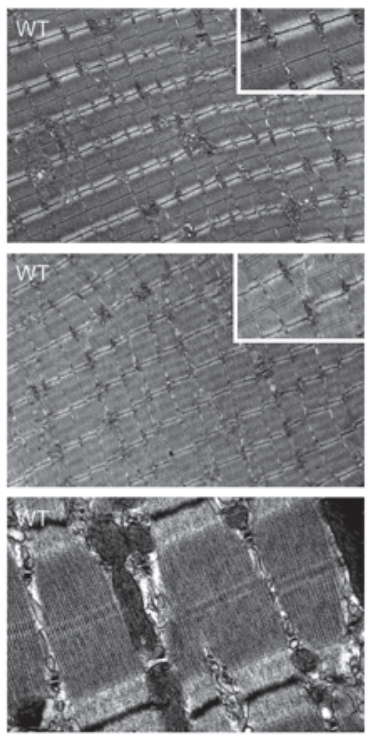
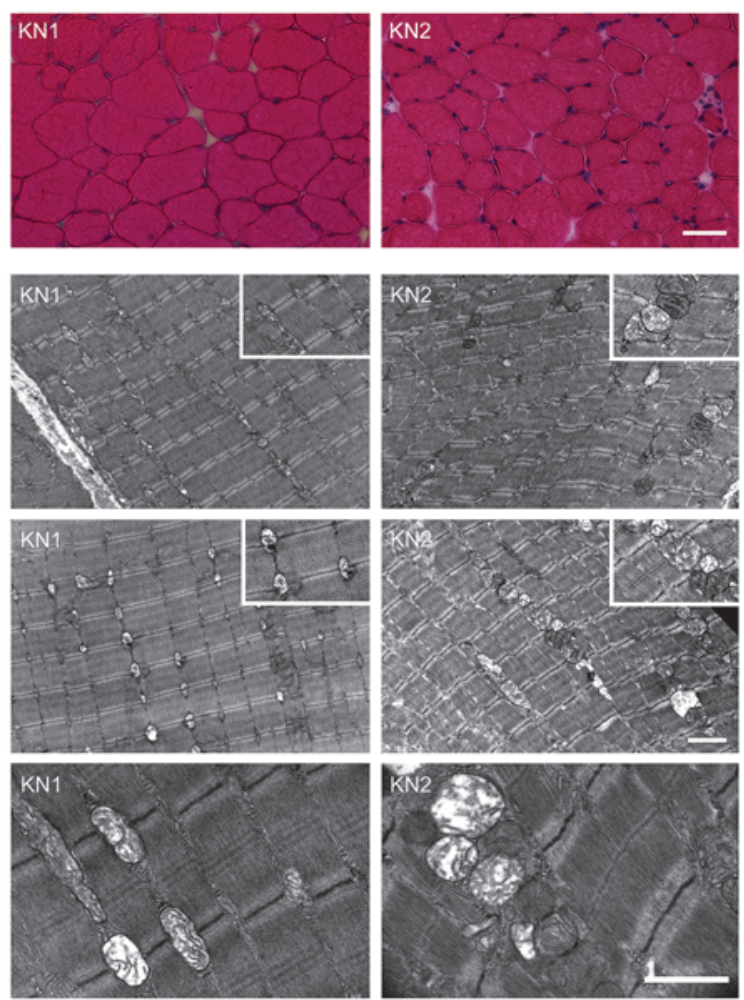

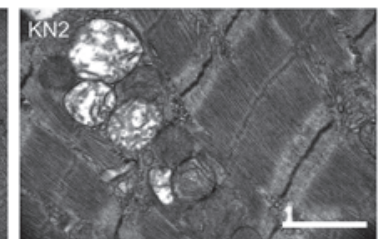

\section{Figure 8}

nNOS splice variant-deficiency leads to myopathic changes in intermyofibrillar mitochondria and skeletal muscle cytoarchitecture. (A) Decreased myofiber CSA was evident in hematoxylin and eosin-stained KN2 TA muscles. (B) Electron microscopic analysis of KN1 muscles revealed dramatic changes in mitochondrion morphology (bottom row), including irregular swelling, disruption of internal cristae, and markedly decreased matrix density (increased electron lucency). Sarcomere integrity and registration were unaffected in KN1 muscle. KN2 muscles exhibited more severe mitochondrion morphological abnormalities, including substantial swelling and variability in size, disrupted cristae, and reduced matrix density. The characteristic intermyofibrillar localization of mitochondria at the Z-I band interface was also disrupted in KN2 muscle (bottom row). Furthermore, loss of all nNOS splice variants often impaired alignment of sarcomere contractile units. Higher-magnification images are shown in the inset panels. Scale bar: $40 \mu \mathrm{m}(\mathbf{A}) ; 2 \mu \mathrm{m}$ (top and middle rows of $\mathbf{B}$ ); $1 \mu \mathrm{m}$ (bottom row of B); $1.3 \mu \mathrm{m}$ (insets). $n=4$.
C57BL/6 KN1 muscles, which also presumably lack normal vasomodulatory controls, exhibit normal fatigue and muscle contractility despite mitochondrial abnormalities. This point is exemplified by $\alpha$-syntrophin-null muscles lacking sarcolemmal nNOS $\mu$ that exhibit aberrant vasoconstriction but show normal fatigue and postexercise strength. In fact, $\alpha$-syntrophin-deficient muscles exhibit no deficits in contractility, consistent with our previous observation that $\alpha$-syntrophin-null mice exhibit normal voluntary wheel running exercise performance (27). Thus, the regulation of fatigue by nNOS $\mu$ is dependent in part on the genetic strain of the mouse. This is not a surprising finding, given that exercise performance, which is dependent in part on muscle performance, varies substantially between mice of different genetic backgrounds (35). It is therefore essential that future studies of muscle fatigue use correct strain controls.

Another potential role for sarcolemmal $\mathrm{nNOS} \mu$ in muscular dystrophy has come from efforts to explain the exaggerated postexercise inactivity symptomatic of dystrophinopathy patients (18). KN1 mice (B6/129 background) and $m d x$ mice (C57BL/10 background), a mouse model for DMD, both exhibited significant postexercise cage inactivity for up to 30 minutes after treadmill running (18). We previously reported that these KN1 mice show increased susceptibility to contraction-induced fatigue and exhibit a slow but full restoration of muscle strength after 5 minutes of rest (10). Thus, claims that the exaggerated fatigue response is distinct from a loss in specific force production by muscle and that KN1 mice have no loss of muscle-specific force after exercise are not supported by the evidence (18). Therefore, early but not prolonged postexercise inactivity in cages may result from excessive exercise-induced skeletal muscle fatigue and impaired postexercise force generation. Furthermore, the normal fatigue resistance and postexercise strength in KN1 and $\alpha$-syntrophin mice in the
C57BL/6 background suggests that contraction-induced sarcolemmal nNOS $\mu$ signaling does not play a significant role in postexercise muscle function in this strain. These data, in conjunction with what we believe are previously unrecognized Golgi nNOS signaling pathways in muscle, demonstrate that the underlying molecular mechanisms of nNOS-modulated postexercise inactivity are more complex than previously thought (18).

In summary, we have demonstrated the existence of what we believe is a novel Golgi nNOS signaling compartment in skeletal muscle and have shown that nNOS splice variants are essential for maintaining the structural health and functional capacity of skeletal muscle tissue. We conclude that nNOS is a critical regulator of skeletal muscle exercise performance.

\section{Methods}

Generation of mutant mice. All experimental procedures performed on mice were approved by the Institutional Animal Care and Use Committee at the University of Washington. Three mutant mouse knockout lines (all on a C57BL/ 6 background) lacking normal nNOS splice variant localization or expression were used (Figure 2, B-E). The first strain is the original nNOS knockout, now known as KN1 (nNOS knockout 1). In the KN1 mouse line, targeted deletion of exon 2 eliminates expression of muscle-specific $\mathrm{nNOS} \mu$ (24); however, the regulatory elements controlling expression of $\mathrm{nNOS} \beta$ and $\mathrm{nNOS} \gamma$ splice variants remain intact (Figure 2D). KN1 mice were backcrossed for 10 generations to the C57BL/6 line. KN2 (nNOS knockout 2) mice were generated by targeted disruption of heme-binding exon 6 (25). KN2 mice do not express any active full-length nNOS splice variants (Figure 2E). The third nNOS mutant mouse line was deficient in $\alpha$-syntrophin and has been previously described (Figure 2C) (27).

Immunohistochemistry. The method used to isolate and immunolabel single myofibers has been described previously (23). Amino and carboxyl terminal pan-specific nNOS rabbit polyclonal antibodies were purchased from 


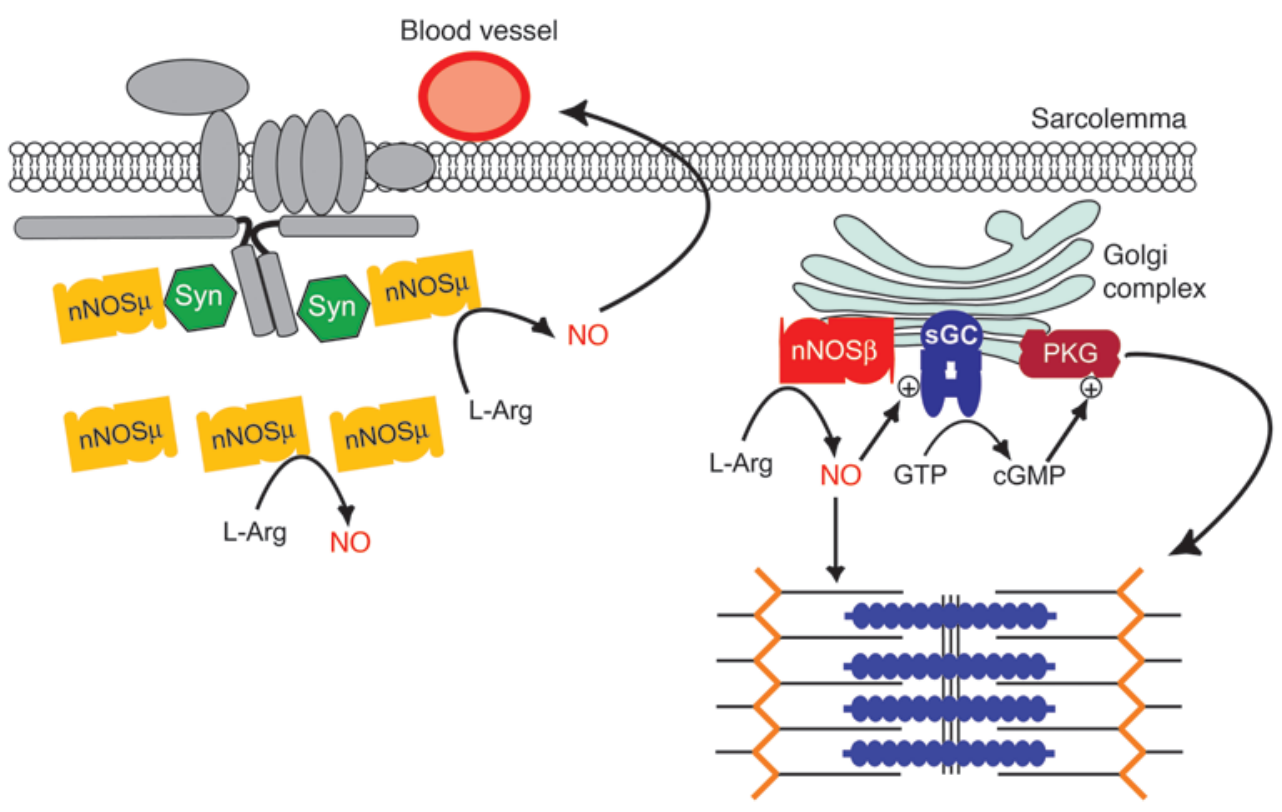

Figure 9

Model for nNOS splice variant microdomain signaling in skeletal muscle. The differential targeting of nNOS $\beta$ and $\mathrm{nNOS} \mu$ creates 2 spatially and functionally distinct NO signaling compartments at the Golgi and sarcolemma, respectively. Spatial confinement of nNOS $\beta$ and its effectors (sGC and PKG) creates a NO-cGMP signaling microdomain at the Golgi complex. During exercise, nNOS $\mu$-derived NO attenuates vasoconstriction, thus serving to match blood and oxygen delivery with the increased metabolic demands of skeletal muscle tissue. At the same time, $\mathrm{nNOS} \beta$ signaling functions to maintain the ability of contracting muscles to generate force during and after exercise. nNOS $\beta$-derived NO may regulate contractility through cGMPdependent mechanisms (mediated by PKG) or by cGMP-independent mechanisms (such as nitrosylation).

Invitrogen (amino terminal), Sigma-Aldrich, BD Biosciences, and Immunostar Inc. (all carboxyl terminal). Rabbit polyclonal antibodies raised against the $\beta 1$ subunit of sGC and FITC-conjugated GM130 were purchased from Sigma-Aldrich and BD Biosciences, respectively. A rabbit polyclonal to PKG was purchased from Santa Cruz Biotechnology Inc. Rabbit polyclonal antibodies were detected with Rhodamine Red-X-conjugated donkey anti-rabbit secondary antibodies (Jackson ImmunoResearch Laboratories Inc.)

Skeletal muscle fiber typing. To characterize the fiber composition of skeletal muscles, $10-\mu \mathrm{m}$-thick frozen sections were immunolabeled with mouse monoclonal antibodies (a gift from Stephen Hauschka, University of Washington) raised against type I (BA-D5), type IIa (SC-71), and type IIb (BF-F3) MyHC proteins (36). Sections were incubated for 1 hour at room temperature with a BA-D5, SC-71, and BF-F3 cocktail. Primary antibodies were detected by a 1-hour incubation with Alexa Fluor 350-labeled donkey anti-mouse IgG2B (BA-D5), Alexa Fluor 594-labeled donkey anti-mouse IgG1 (SC-71), and Alexa Fluor 488 donkey anti-mouse IgM (BF-F3) isotype-specific secondary antibodies. The frequency of MyHC type I, type IIa, type IIb, and type IIx/IId fibers per section were counted manually. Unlabeled fibers were designated type IId/IIx. The area of the muscle section in square millimeters was used to calculate the number of $\mathrm{MyHC}$ fibers per square millimeter, using IMAGE J V1.38x software (37).

Confocal laser scanning microscopy. Confocal microscopy was performed at the W.M. Keck Center for Advanced Studies in Neural Signaling (University of Washington), using a Leica TCS SP microscope equipped with a UV laser used to excite DAPI.

Tissue histology and myofiber CSA calculations. Freshly isolated mouse TA and soleus muscles were isolated from adult (8- to 9-week-old) mice and flash frozen in liquid nitrogen-cooled isopentane. Cryostat sections $(10 \mu \mathrm{m})$ from the muscle mid-belly were stained with hematoxylin and eosin using standard methods and inspected for signs of muscle disease, including central nucleation, fiber area heterogeneity, and immune cell infiltration. Histological analyses were performed with a Zeiss Axioskop 2 microscope. The CSA of more than 200 muscle cells from a section (3-5 sections were analyzed per mouse) of the muscle mid-belly was measured using ImageJ V1.38x software (http:// rsbweb.nih.gov/ij/) (37).

Transmission electron microscopy. Skeletal muscle tissue was fixed in half-strength Karnovsky's fixative (2.0\% paraformaldehyde, $2.5 \%$ glutaraldehyde, $0.1 \mathrm{M}$ cacodylate buffer, $3 \mathrm{mM} \mathrm{CaCl}_{2}, \mathrm{pH} 7.3$ ), post-fixed in $1.0 \% \mathrm{OsO}_{4}$ for 1 hour, en bloc stained with $2 \%$ uranyl acetate, and rinsed and then dehydrated through a graded series of alcohols and propylene oxide. Samples were embedded with Eponate resin (Ted Pella Inc.), and 90- to $100-\mathrm{nm}$ sections were cut and mounted for transmission electron microscopy examination on 150 mesh rhodium/copper grids and stained with uranyl acetate and lead citrate. Samples were examined with a JEM 1200EX II transmission electron microscope (JEOL Ltd.). Operating conditions for the instrument included an accelerating voltage of $80 \mathrm{kV}$, a $300 \mu \mathrm{m}$ condenser aperture, a $50 \mu \mathrm{m}$ objective aperture, and a spot size setting of 4. Images were acquired and saved digitally using an Olympus-SIS Morada side mount camera (Olympus Soft Imaging Solutions).

In situ analysis of skeletal muscle contractile function. All in situ tests of muscle contractile performance were identical to those described previously (10). The advantage of in situ analysis is that vascularization and vasomodulatory controls remain intact. This is an important consideration when assessing nNOS splice variant function in skeletal muscle, given the important role of $\mathrm{nNOS} \mu$ in regulating blood delivery during muscle contraction and the dependence of NO function on oxygen concentration $(7,8,38)$. To test muscle resistance to fatigue, TA muscles were maximally stimulated $(40 \mathrm{~V}$, $200 \mathrm{~Hz}$ ) every 2 seconds for 4 minutes to simulate exercise-induced fatigue. Maximum isometric force production was recorded every 2 seconds during exercise and at 1 minute and 5 minutes after exercise. Exponential curves $\left(y=A e^{-x / \tau}\right.$, where $A$ is the initial force in millinewtons, $x$ is time in seconds, and $\tau$ is the time constant) were fitted to the 4-minute fatigue period with Igor Pro 5 software (Wavemetrics). The time constant, which reflects the time taken to fatigue, and the force plateau (the asymptote of the exponential curve) were calculated from these fitted curves.

Serum creatine kinase assay. Blood $(20 \mu \mathrm{l})$ was collected from adult 8-weekold mice, allowed to clot for 20 minutes, and then microcentrifuged to separate the serum. Serum creatine kinase levels were determined using a commercially available kit, according to the manufacturer's protocol (Stanbio Laboratory). Serum creatine kinase activity levels reflect whole muscle cell structural stability. Muscle cell turnover or breakdown leads to the release of cytosolic enzymes, including creatine kinase, into the bloodstream. 
Statistics. All values, except CSAs, are reported as mean \pm SEM. For normally distributed populations, statistically significant differences between 3 or more groups were tested for with 1-way ANOVA, followed by the Tukey-Kramer multiple comparison post-hoc tests between paired groups. CSA measurements made on hematoxylin and eosin sections did not account for fiber type-specific differences in size and thus did not conform to a Gaussian distribution and failed the Kolmogorov-Smirnov test for normality. In order to test for significant differences between CSAs, group medians were compared with nonparametric Kruskal-Wallis ANOVA by ranks, followed by Dunn's multiple comparison post-hoc test. Statistical calculations were performed using Prism version 4 software (Graphpad Software Inc.). $P$ values of less than 0.05 were considered significant.

\section{Acknowledgments}

We thank Greg Martin of the Keck Imaging Center at the University of Washington for microscopy assistance, the Hauschka laboratory for assistance with myosin immunohistochemistry, and Stephanie Lara and Steve MacFarlane for assistance with electron microscopy. We thank Kimberley Craven, Michael Puljung, and members of the Froehner laboratory for manuscript editing and advice. This work was supported by grants from the Muscular Dystrophy Association (to J.M. Percival), NIH (to S.C. Froehner), and National Institute of Neurological Disorders and Stroke (to P.L. Huang).

Received for publication August 6, 2009, and accepted in revised form December 9, 2009.

Address correspondence to: Justin M. Percival, Department of Physiology and Biophysics, University of Washington, Box 357290, Seattle, WA 98195-7290, USA. Phone: 206.543.9094; Fax: 206.685.0619; E-mail: justinp2@u.washington.edu.
1. Bredt DS, Snyder SH. Isolation of nitric oxide synthetase, a calmodulin-requiring enzyme. Proc Nat Acad Sci U S A. 1990;87(2):682-685.

2. Silvagno F, Xia H, Bredt DS. Neuronal nitric-oxide synthase-mu, an alternatively spliced isoform expressed in differentiated skeletal muscle. J Biol Chem. 1996;271(19):11204-11208.

3. Brenman JE, Chao DS, Xia H, Aldape K, Bredt DS. Nitric oxide synthase complexed with dystrophin and absent from skeletal muscle sarcolemma in Duchenne muscular dystrophy. Cell. 1995;82(5):743-752.

4. Brenman JE, et al. Interaction of nitric oxide synthase with the postsynaptic density protein PSD-95 and alpha1-syntrophin mediated by PDZ domains. Cell. 1996;84(5):757-767.

5. Stamler JS, Meissner G. Physiology of nitric oxide in skeletal muscle. Physiol Rev. 2001;81(1):209-237.

6. McConell GK, et al. Skeletal muscle nNOS mu protein content is increased by exercise training in humans. Am J Physiol Regul Integr Comp Physiol. 2007;293(2):R821-R828.

7. Thomas GD, Shaul PW, Yuhanna IS, Froehner SC, Adams ME. Vasomodulation by skeletal musclederived nitric oxide requires alpha-syntrophinmediated sarcolemmal localization of neuronal nitric oxide synthase. Circ Res. 2003;92(5):554-560.

8. Thomas GD, et al. Impaired metabolic modulation of alpha-adrenergic vasoconstriction in dystrophin-deficient skeletal muscle. Proc Natl Acad Sci US A. 1998;95(25):15090-15095.

9. Ross RM, Wadley GD, Clark MG, Rattigan S, McConell GK. Local nitric oxide synthase inhibition reduces skeletal muscle glucose uptake but not capillary blood flow during in situ muscle contraction in rats. Diabetes. 2007;56(12):2885-2892.

10. Percival JM, Anderson KN, Gregorevic P, Chamberlain JS, Froehner SC. Functional deficits in nNOSmu-deficient skeletal muscle: myopathy in nNOS knockout mice. PLoS ONE. 2008;3:e3387.

11. Lancaster JR Jr. A tutorial on the diffusibility and reactivity of free nitric oxide. Nitric Oxide. 1997;1(1):18-30.

12. Saraiva RM, Hare JM. Nitric oxide signaling in the cardiovascular system: implications for heart failure. Curr Opin Cardiol. 2006;21(3):221-228.

13. Flögel U, Merx MW, Godecke A, Decking UK, Schrader J. Myoglobin: A scavenger of bioactive NO. Proc Natl Acad Sci U S A. 2001;98(2):735-740.
14. Barouch LA, et al. Nitric oxide regulates the heart by spatial confinement of nitric oxide synthase isoforms. Nature. 2002;416(6878):337-339.

15. Jaffrey SR, Snowman AM, Eliasson MJ, Cohen NA, Snyder SH. CAPON: a protein associated with neuronal nitric oxide synthase that regulates its interactions with PSD95. Neuron. 1998;20(1):115-124.

16. Chao DS, et al. Selective loss of sarcolemmal nitric oxide synthase in Becker muscular dystrophy. J Exp Med. 1996;184(2):609-618.

17. Crosbie RH, Barresi R, Campbell KP. Loss of sarcolemma nNOS in sarcoglycan-deficient muscle. FASEB J. 2002;16(13):1786-1791.

18. Kobayashi YM, et al. Sarcolemma-localized nNOS is required to maintain activity after mild exercise. Nature. 2008;456(7221):511-515.

19. Wehling M, Spencer MJ, Tidball JG. A nitric oxide synthase transgene ameliorates muscular dystrophy in mdx mice. J Cell Biol. 2001;155(1):123-131.

20. Lai Y, et al. Dystrophins carrying spectrin-like repeats 16 and 17 anchor nNOS to the sarcolem$\mathrm{ma}$ and enhance exercise performance in a mouse model of muscular dystrophy. J Clin Invest. 2009; 119(3):624-635.

21 . Brunelli $\mathrm{S}$, et al. Nitric oxide release combined with nonsteroidal antiinflammatory activity prevents muscular dystrophy pathology and enhances stem cell therapy. Proc Natl Acad Sci U S A. 2007; 104(1):264-269.

22. Colussi C, et al. HDAC2 blockade by nitric oxide and histone deacetylase inhibitors reveals a common target in Duchenne muscular dystrophy treatment. Proc Natl Acad Sci U S A. 2008;105(49):19183-19187.

23. Percival JM, et al. rAAV6-microdystrophin rescues aberrant Golgi complex organization in $\mathrm{mdx}$ skeletal muscles. Traffic. 2007;8(10):1424-1439.

24. Huang PL, Dawson TM, Bredt DS, Snyder SH, Fishman MC. Targeted disruption of the neuronal nitric oxide synthase gene. Cell. 1993;75(7):1273-1286.

25. Gyurko R, Leupen S, Huang PL. Deletion of exon 6 of the neuronal nitric oxide synthase gene in mice results in hypogonadism and infertility. Endocrinology. 2002;143(7):2767-2774.

26. Thyberg J, Moskalewski S. Microtubules and the organization of the Golgi complex. Exp Cell Res. 1985; 159(1):1-16.

27. Adams ME, et al. Absence of alpha-syntrophin leads to structurally aberrant neuromuscular synapses defi- cient in utrophin. J Cell Biol. 2000;150(6):1385-1398.

28. Larsson L, Edström L, Lindegren B, Gorza L, Schiaffino S. MHC composition and enzyme-histochemical and physiological properties of a novel fast-twitch motor unit type. Am J Physiol. 1991; 261(1 Pt 1):C93-C101.

29. Morris RL, Hollenbeck PJ. Axonal transport of mitochondria along microtubules and F-actin in living vertebrate neurons. J Cell Biol. 1995; 131(5):1315-1326.

30. Pisconti A, et al. Follistatin induction by nitric oxide through cyclic GMP: a tightly regulated signaling pathway that controls myoblast fusion. J Cell Biol. 2006;172(2):233-244.

31. Hao G, Derakhshan B, Shi L, Campagne F, Gross SS. SNOSID, a proteomic method for identification of cysteine S-nitrosylation sites in complex protein mixtures. Proc Natl Acad Sci U S A. 2006; 103(4):1012-1017.

32. Erusalimsky JD, Moncada S. Nitric oxide and mitochondrial signaling: from physiology to pathophysiology. Arterioscler Thromb Vasc Biol. 2007; 27(12):2524-2531.

33. Tidball JG, Wehling-Henricks $M$. The role of free radicals in the pathophysiology of muscular dystrophy. J Appl Physiol. 2007;102(4):1677-1686.

34. Sander M, et al. Functional muscle ischemia in neuronal nitric oxide synthase-deficient skeletal muscle of children with Duchenne muscular dystrophy. Proc Natl Acad Sci U S A. 2000;97(25):13818-13823.

35. Lerman I, et al. Genetic variability in forced and voluntary endurance exercise performance in seven inbred mouse strains. J Appl Pbysiol. 2002;92(6):2245-2255.

36. Salva MZ, et al. Design of tissue-specific regulatory cassettes for high-level rAAV-mediated expression in skeletal and cardiac muscle. Mol Ther. 2007; 15(2):320-329.

37. Abramoff MD, Magelhaes PJ, Ram SJ. Image processing with Image J. Biophotonics International. 2004; $11: 36-42$

38. Eu JP, et al. Concerted regulation of skeletal muscle contractility by oxygen tension and endogenous nitric oxide. Proc Natl Acad Sci U S A. 2003; 100(25):15229-15234.

39. Brenman JE, Xia H, Chao DS, Black SM, Bredt DS. Regulation of neuronal nitric oxide synthase through alternative transcripts. Dev Neurosci. 1997; 19(3):224-231. 